\title{
Molecular-dynamics simulations of liquid aluminum oxide
}

\author{
Miguel Angel San Miguel and Javier Fernández Sanz \\ Departamento de Química Física, Facultad de Química, E-41012, Sevilla, Spain \\ Luis Javier Álvarez \\ Laboratorio de Simulación de Materiales, Dirección General de Servicios de Cómputo Académico, Universidad Nacional Autónoma de \\ México, Insurgentes Sur No. 3000, Zona Cultural, Ciudad Universitaria, Coyoacán 04510, México D. F., Mexico \\ José Antonio Odriozola \\ Departamento de Química Inorgánica e Instituto de Ciencia de Materiales, Universidad de Sevilla-CSIC, E-41012 Sevilla, Spain
}

(Received 20 April 1998)

\begin{abstract}
The total and partial radial distribution functions $g(r)$ and the corresponding structure factors $S(q)$ were calculated based on molecular-dynamics simulations in the microcanonical ensemble of liquid aluminum oxide. The simulations were performed in the temperature range $2300-3000 \mathrm{~K}$ in order to explore the temperature dependence of the structure, finding that the liquid structure is invariant as a function of temperature. With the aid of the partial radial distribution functions, it is possible to reinterpret the experimental data leading to a new assignment of peaks which differs from the one reported in a previously published work by Ansell et al. [Phys. Rev. Lett. 78, 464 (1997)]. The structural model for liquid aluminum oxide obtained from our simulations is essentially the same as that reported in the experimental work. [S0163-1829(98)01530-6]
\end{abstract}

The determination of the structure of materials has always been subject to interpretation of experimental data. With the advent of computer simulation methods it has been possible to refine the assignment of peaks of radial distribution functions to interacting pairs of particles. Furthermore, numerical simulation allows one to avoid experimental difficulties such as having samples at extreme thermodynamic conditions. Aluminum oxide is one of the most important ceramic materials due to the great variety of technological applications. ${ }^{2}$ It has a wide variety of metastable crystalline phases which are classified as $\alpha$ and $\gamma$ series. Extensive simulation studies of the structure and dynamics of $\gamma$-alumina have been reported and the interpretation of its structure has been approached correlating simulation results with different experimental techniques. ${ }^{3}$ Recently a report of the experimental determination of the structure of liquid aluminum oxide has been published. ${ }^{1}$ In order to complement this experiment, in this work we report the results of molecular-dynamics (MD) simulations of liquid aluminum oxide, and discuss a model to describe its structure.

The simulations were performed in the microcanonical ensemble with a system consisting of 960 particles of which 384 represented aluminum atoms and 576 oxygen atoms arranged in a cubic box. The pair interaction potential is a Pauling-type function given by

$$
V_{i j}\left(r_{i j}\right)=\frac{q_{i} q_{j}}{r_{i j}}+\frac{1}{n\left(\theta_{i}+\theta_{j}\right)}\left(\frac{\theta_{i}+\theta_{j}}{r_{i j}}\right)^{n}
$$

where $q_{i}$ are effective charges, $2.325 e$ for aluminum atoms and $-1.55 e$ for oxygen atoms; $\theta_{i}$ are the atomic radii taken from Shannon and Prewit ${ }^{4}$ and $n$ was taken to be 9 as was proposed by Adams and $\mathrm{McDonald}^{5}$ for ionic systems. The long-range coulombic interactions were calculated using the Ewald summation method. ${ }^{6}$
In order to make the simulated system comparable with the experiment reported in Ref. 1, we started with a solid corundum structure in a computational box with periodic boundary conditions, whose volume was set $20 \%$ higher than the normal to account for the volume expansion reported to occur upon melting. ${ }^{7}$ In order for the system to achieve the liquid disordered state, several different procedures were tested. All of them yielded the same structural rearrangements and what we report here is the result of having taken the $\alpha$-alumina system into the cubic box in a first run at 300 $\mathrm{K}$, in which the system just expanded to fill the computational box but there were no considerable structural changes. Then a long simulation of $100 \mathrm{ps}$ at $2260 \mathrm{~K}$ was performed rescaling the velocities of all particles every time step. From this stage, five simulations were carried out in the temperature range $2260-2990 \mathrm{~K}$. All of them consisted of a first run of $10 \mathrm{ps}$ with temperature control; a second one of other 10 ps in which the system was free to evolve in order to assure the thermodynamic equilibrium; and a final one of $100 \mathrm{ps}$ to calculate statistical averages. The integration of the classical equations of motion was carried out using the leap-frog algorithm with an integration time step of $1 \mathrm{fs}$. Energy fluctuations were of $0.01 \mathrm{~kJ} / \mathrm{mol}$ around the mean value although there was a temperature drift in the equilibration runs of plus or minus $40 \mathrm{~K}$.

Figure 1 shows the total radial distribution functions of the simulated system at 2260 and $2990 \mathrm{~K}$. Figure 2 shows the structure factor $S(q)$ for the system at $2260 \mathrm{~K}$ obtained by Fourier transforming the corresponding $g(r)$. For the sake of comparison, the experimental results reported by Ansell et al. ${ }^{1}$ have also been included in these figures. The two curves corresponding to liquid alumina in Fig. 1 are quite similar and have the same features as those for the supercooled liquid $\mathrm{Al}_{2} \mathrm{O}_{3}$ and stable liquid $\mathrm{Al}_{2} \mathrm{O}_{3}$ obtained experimentally. However, as we shall see below, the assign- 


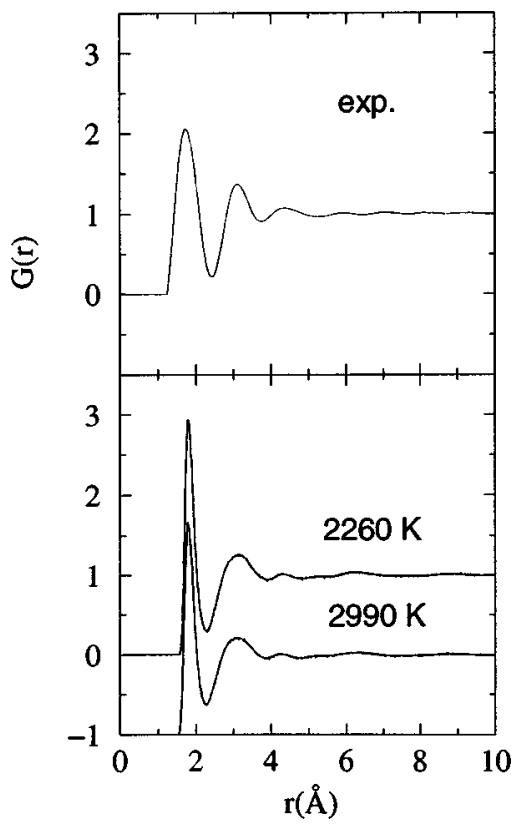

FIG. 1. Total radial distribution functions for liquid $\mathrm{Al}_{2} \mathrm{O}_{3}$. Top: experimental spectrum at $2223 \mathrm{~K}$ (Ref. 1). Bottom: computed from MD simulations at 2260 and $2990 \mathrm{~K}$. The values of the lower curve have been shifted by 1 for clarity.

ment of the radial distribution functions maxima differs from the interpretation reported in that work. As it can be observed both functions are in excellent agreement with the experimental data, including the fact that in the temperature range $2260-2990 \mathrm{~K}$ the shape of the curves is invariant as a function of temperature. The first three peaks were assigned by Ansell et al. ${ }^{1}$ to the $\mathrm{Al}-\mathrm{O}, \mathrm{O}-\mathrm{O}$, and $\mathrm{O}-\mathrm{O}$ next-nearest-

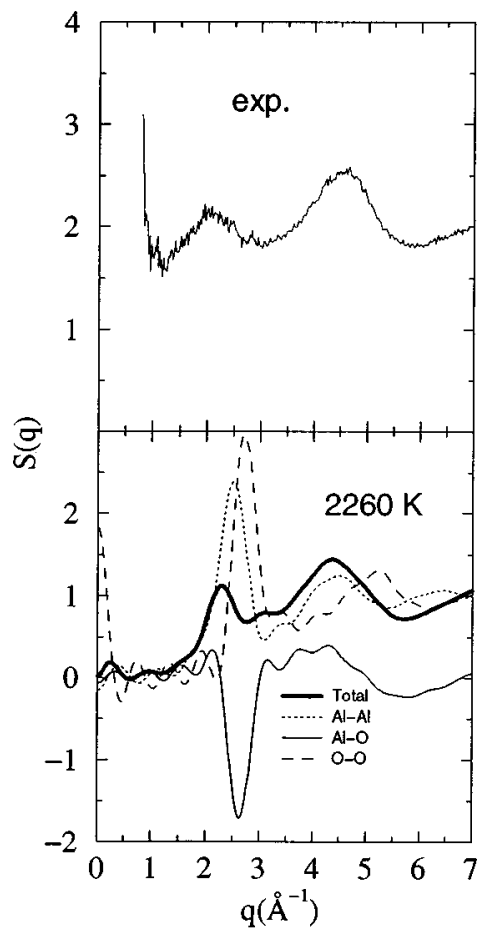

FIG. 2. Structure factor $S(q)$ for liquid $\mathrm{Al}_{2} \mathrm{O}_{3}$. Top: experimental data at $2223 \mathrm{~K}$ (Ref. 1). Bottom: computed from MD simulations at $2260 \mathrm{~K}$ by Fourier transforming the corresponding $g(r)$.

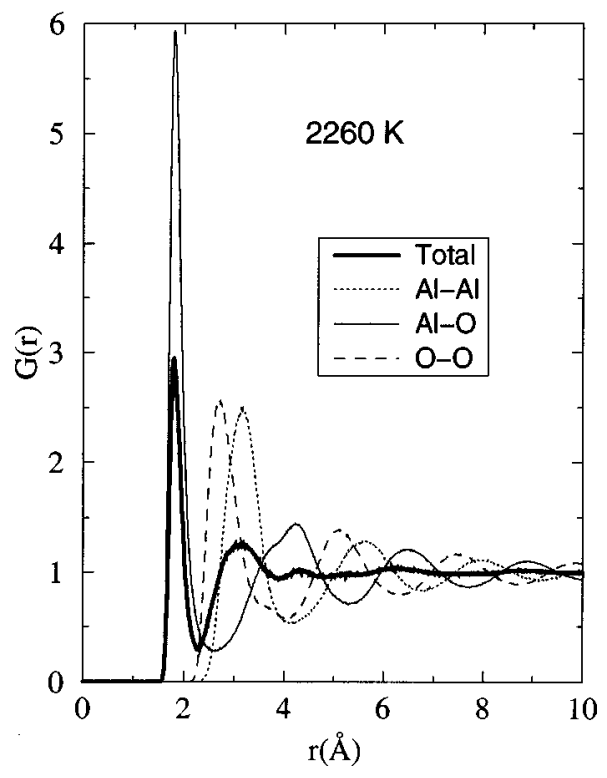

FIG. 3. Partial radial distribution functions.

neighbor pairs, respectively. However, looking at the partial radial distribution functions shown in Fig. 3, it is very easy to see the pairs which produce the main peaks of the total radial distribution function. The first peak, at $1.76 \AA$, is clearly due to the first coordination shell of $\mathrm{Al}$, in agreement with the assignment of Ansell et al. The second one, at $2.9 \AA$ in our simulations, and $3.08 \AA$ the experimental, is a superposition of the $\mathrm{Al}-\mathrm{Al}$ and $\mathrm{O}-\mathrm{O}$ closest distances and not only to the O-O pair. Finally, the third peak, at $4.25 \AA$, is due to the second coordination shell of $\mathrm{Al}$, and not to the O-O nextnearer-neighbor correlation.

The coordination number of $\mathrm{Al}$ atoms, obtained from the integration of the Al-O partial radial distribution functions is 4.4. This result agrees with that reported in Ref. 1 of 4.4 \pm 1.0. The ${ }^{27} \mathrm{Al}$ NMR spectrum of $\mathrm{Al}_{2} \mathrm{O}_{3}$ liquid shows a

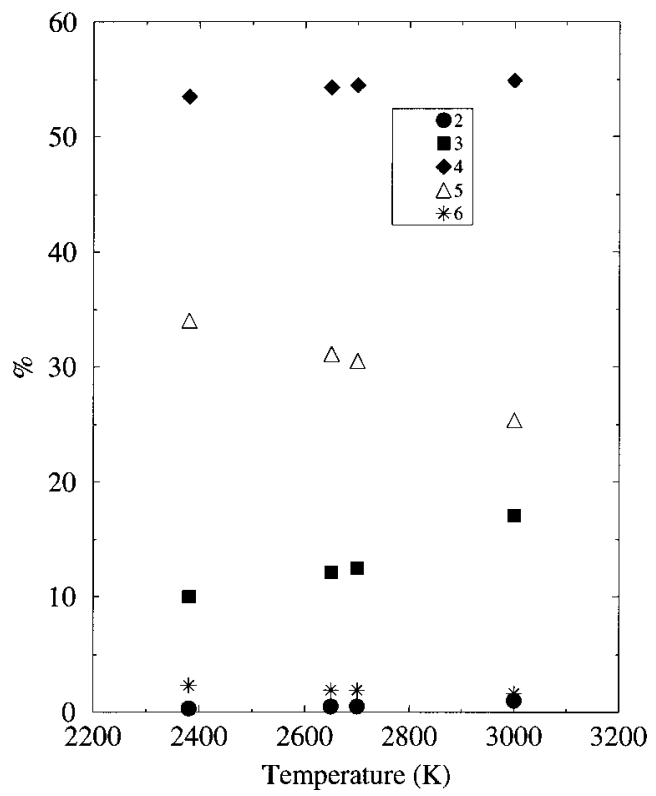

FIG. 4. Percentage of $\mathrm{Al}$ atoms for every coordination number at four different temperatures. 


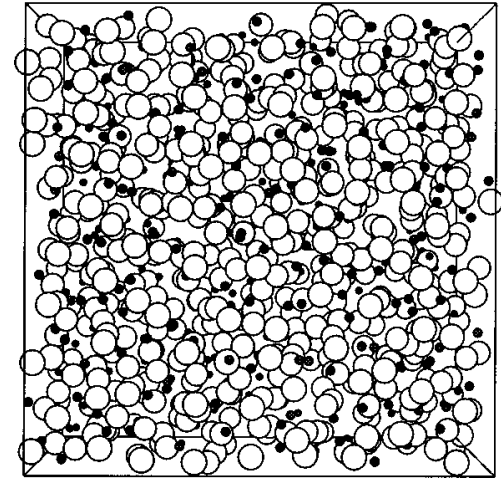

FIG. 5. Snapshot of the system in the liquid state.

single line with a chemical shift of $59 \mathrm{ppm}^{8}$ This value is between the ones corresponding to four- and six-coordinated $\mathrm{Al}$ sites. Coutures et al. ${ }^{9}$ originally proposed the presence of tetrahedral and octahedral aluminum atoms undergoing fast exchange and yielding an average coordination number of 4.5 atoms. Later on, Poe et al. ${ }^{10}$ suggested the presence of five-coordinated Al sites in the liquid. Our simulations indicate that low coordinated $\mathrm{Al}$ and the average coordination number is around 4.5 atoms in a wide range of temperatures.

Figure 4 presents the percentage of $\mathrm{Al}$ atoms for every coordination number at four different temperatures. The four-coordinated aluminum atoms are the most abundant, between 53.5 and $55 \%$, and increase very little with increasing temperature. The percentage of five-coordinated $\mathrm{Al}$ atoms diminishes with increasing temperature. This tendency is observed in the six-coordination case, whereas it is the opposite in the three-coordinated $\mathrm{Al}$ atoms. These are the ones which change the most with temperature at the expense of the fivecoordinated $\mathrm{Al}$ atoms. When temperature increases, some of the five-coordinated $\mathrm{Al}$ go to coordination four and some of them to coordination three.

Figure 5 shows a snapshot of the system in the liquid state, from which there were extracted three pictures of representative polyhedra found in the simulated liquid, shown in Fig. 6. In the latter, the top view is a model of the most common situation and presumably the most stable in the system. This model is not stoichiometric, it has an excess of oxygen atoms, therefore the remaining $\mathrm{Al}$ atoms should either be low-coordinated or should form edge-sharing polyhedra as can be seen in the bottom views in Fig. 6. The bridg-
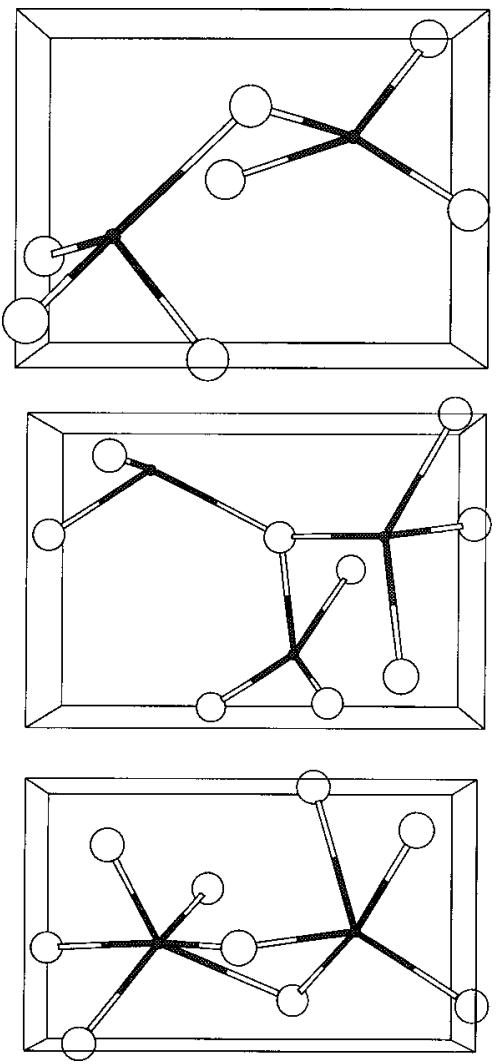

FIG. 6. Pictures of representative polyhedra found in the simulated liquid.

ing oxygen atom in the model appears as a two-coordinated atom, but in general oxygen atoms appear connected to a third low-coordination polyhedron.

Given the excellent agreement between the simulated and experimental results one can conclude that the interpretation of experimental data can be refined based on our simulations. The structural changes observed in the simulated system, including the coordination changes, are reproduced by our simulations. With the aid of the partial radial distribution functions it is possible to identify the pairs which are responsible for the peaks of the experimental total radial distribution function.

This work was supported by the DGICYT (SPAIN, Project No. PB95-1247). M.A.S. thanks the Ministerio de Educación y Ciencia for the award of a FPI grant.
${ }^{1}$ S. Ansell, S. Krishnan, J. K. R. Weber, J. J. Felten, P. C. Nordine, M. A. Beno, D. L. Price, and M-L. Saboungi, Phys. Rev. Lett. 78, 464 (1997).

${ }^{2}$ E. Dorre, Alumina: Processing Properties and Applications (Springer-Verlag, New York, 1984).

${ }^{3}$ L. J. Álvarez, J. Fernández Sanz, M. J. Capitán, and J. A. Odriozola, Chem. Phys. Lett. 192, 463 (1992); L. J. Álvarez, L. E. León, J. Fernández Sanz, M. J. Capitán, and J. A. Odriozola, Phys. Rev. B 50, 2561 (1994); J. Phys. Chem. 99, 17872 (1995); L. J. Álvarez, A. L. Blumenfeld, and J. J. Fripiat, J. Chem. Phys. (to be published)

${ }^{4}$ R. D. Shannon and C. T. Prewitt, Acta Crystallogr., Sect. B:
Struct. Crystallogr. Cryst. Chem. 25, 925 (1969).

${ }^{5}$ D. J. Adams and I. R. McDonald, Physica B 79, 159 (1970).

${ }^{6}$ P. P. Ewald, Ann. Phys. (Leipzig) 64, 253 (1921).

${ }^{7}$ P. Tyrolerova and W. K. Lu, J. Am. Chem. Soc. 52, 77 (1969).

${ }^{8}$ R. K. Sato, P. F. McMillan, P. Dennison, and R. Dupree, J. Phys. Chem. 95, 4483 (1991).

${ }^{9}$ J-P. Coutures, D. Massiot, C. Bessada, P. Echegut, J-C. Rifflet, and F. Taulelle, C. R. Acad. Sci., Ser. II: Mec., Phys., Chim., Sci. Terre Univers. 310, 1041 (1990).

${ }^{10}$ B. T. Poe, P. F. McMillan, B. Cote, D. Massiot, and J-P. Coutures, J. Phys. Chem. 96, 8220 (1992). 